\title{
The Phase-out of Perfluorooctane Sulfonate (PFOS) and the Global Future of Aqueous Film Forming Foam (AFFF), Innovations in Fire Fighting Foam
}

\author{
Anant R. Sontake', Sameer M.Wagh, ${ }^{2, *}$ \\ ${ }^{1}$ Fire Service Department, Bhilai Steel Plant (SAIL), Bhilai. India \\ ${ }^{2}$ Department of Chemical Technology, Laxminarayan Institute of Technology, RTMNU, Nagpur, India \\ *Corresponding author: vansam2522@rediffmail.com
}

Received January 24, 2014; Revised February 18, 2014; Accepted February 23, 2014

\begin{abstract}
Aqueous film-forming foams (AFFFs) are among the most popular fire-fighting foams used in liquid fuel fires because of their film forming and fast knock down property. One key ingredient of AFFFs, the fluorocarbon surfactant i.e. perfluorooctane sulfonate (PFOS) which is used to reduce surface tension and positive spreading coefficient, is toxic to aquatic life and is a persistent chemical that accumulates in the blood of humans and other animals. Surfactants are not found naturally in the environment and are man-made. In the year 2000 unexpectedly announcement of phasing out fluorocarbon surfactant's manufacturing and its storage which effected a number of product lines, including the firefighting foams. Internationally the manufacturing and release of PFOS to the environment will be suspended by 2015. New fluorosurfactants have been introduced into the market with reformulation and used to form aqueous fire-fighting foam concentrates. The toxicity of the new fluorosurfactants and their persistence in the environment are not well established and still are under investigation. Their presence in the future market is unsure. The continuous research and development to find out the substitute for perfluorooctane sulfonate derivative (C8) has brought two choices i.e. Fluorine-free foams or Fluorotelomer (C6)-based Foams. These foams which may fulfill requirement of different international standards of fire fighting but still contain small amounts of fluorochemicals and are thus not truly fluorine-free. There is every possibility that even after 2015 new regulation may come in to effect to restrict the use of these new formulations (C6) of fire fighting foam. Therefore, the fire-fighting industry has an urgent need for new, environmental friendly foaming agents and foam stabilizers to replace fluorosurfactants in aqueous fire-fighting foams with enhanced drain time, low bubble coarsening, and faster knockdown and excellent burn back resistance properties.
\end{abstract}

Keywords: fire fighting, surfactant, PFOS, phase out, aqueous film-forming foams, surface tension, spreading coefficient

Cite This Article: Anant R. Sontake, and Sameer M.Wagh, "The Phase-out of Perfluorooctane Sulfonate (PFOS) and the Global Future of Aqueous Film Forming Foam (AFFF), Innovations in Fire Fighting Foam.” Chemical Engineering and Science, vol. 2, no. 1 (2014): 11-14. doi: 10.12691/jcsa-2-1-3.

\section{Introduction}

Firefighting foam is an extinguishing agent commonly used for Class B fires i.e. flammable liquid fires. There are many foam concentrates developed with different properties for different applications. Foam form a blanket on the surface of flaming liquid. The blanket prevents flammable vapors from leaving the surface of fuel and prevents oxygen from reaching the fuel. In addition, the water in the foam also has a cooling effect, which gives foam the ability to cool surrounding structure to help prevent burn back. Commercial Class B fire fighting foams are used in many fires mitigation system including system designed for protection of chemical tankers, large petrochemical tanks, heavy hazardous process industries like petroleum, chemical, fertilizer, aircraft and steel industry.
Aqueous film-forming foams (AFFFs) have been commercially available for firefighting from their development by United Sate Navy and 3M Co. in the mid 1960.Since then AFFFs are among the most popular firefighting foams used against fuel and oil fires because of their effectiveness and their ease of application. Unfortunately, some studies have shown that one key ingredient of AFFFs, the fluorocarbon surfactant i.e. perfluorooctane sulfonate (PFOS), is toxic to aquatic life and is a persistent chemical that accumulates in the blood of humans and other animals. On May 16, 2000 3M unexpectedly announced it was phasing out fluorocarbon surfactant manufacturing which effected a number of product lines, including the firefighting foams. The firefighting industry currently is stocked with materials that have been phased out and that, sooner or later, need to be replaced. In 2006 the United States Environmental Protection Agency (USEPA) initiated a voluntary 
Stewardship program to suspend the manufacturing and release of PFOS to the environment by 2015. New fluorosurfactants have been introduced into the market with reformulation and used to formulate aqueous firefighting foam concentrates. The toxicity of the new fluorosurfactants and their persistence in the environment are not well established and still are under investigation. Their presence in the future market is unsure. Therefore, the fire-fighting industry has an urgent need for new, environmentally friendly foaming agents and foam stabilizers to replace fluorosurfactants in aqueous firefighting foams.

\section{Formulation of AFFF}

The formulations of $6 \%, 3 \%$, and $1 \%$ AFFF concentrates are based on performance, not on the constituents. Globally most widely used foam specifications are Underwriters Laboratories Inc. (UL) "Foam Equipment and Liquid Concentrates" (UL 162), U.S. Military Specification (MIL SPEC, MIL-F24385),ISO7203-1 and EN 1568-3 for AFFF. These specifications require that certain performance requirements on medium scale fire along with some physical \& chemical tests. Mil-F-Spec 24385F are more stringent and invites more tests viz;

Table 1. Fofferent test of Foam compound

\begin{tabular}{|l|l|}
\hline 1. Viscosity & 2. Stability \\
\hline 3. Refractive Index & 4. Compatibility \\
\hline 5. Fluorine Content & 6. Dry Chemical Compatibility \\
\hline 7. pH & 8. Film Formation \\
\hline 9. Total Halides & 10. Toxicity \\
\hline $\begin{array}{l}\text { 11. Corrosion- general and } \\
\text { localized }\end{array}$ & 12. Biological Oxygen Demand (BOD) \\
\hline 13. Sealability & 14. Chemical Oxygen Demand (COD) \\
\hline 15. Spreading Coefficient & \\
\hline
\end{tabular}

The foam solution that is formed generally contains the following:

- Water, 98\%-99\%

- Surfactants (fluorosurfactants and hydrocarbon surfactants), 0.03\%-0.45\%

- Butyl Carbitol (glycol ether), 0.5\%-1.1\%

- Ethylene Glycol (not in all formulations), 0.34\%$0.60 \%$

- Urea (not in all formulations), 0.2\%-0.4\%

\section{What is Surfactant?}

Surfactants are not found naturally in the environment and are man-made. They are used in many types of products where their capacity to form smooth water, oil, grease, and stain repellent surfaces is desirable. Metal plating and fire-fighting foams (AFFF) were simulated to contribute to approximately $71 \%$ and $25 \%$, respectively, to the emissions of PFOS, where as emissions from photography (2\%), photolithography and semiconductors ( $1 \%)$, textile protection $(0.4 \%)$, as well as paints and lacquers $(0.2 \%)$ contributed to smaller extents.

Various surfactants are used in foam concentrates to reduce surface tension and positive spreading coefficient that enables film formation on top of the lighter fuel. An example of a common fluorinated surfactant is the sodium or potassium salt of perfluorooctaneulfonate derivative, C8F17SO2-R-COONa or C8F17SO2-R-COOK, consisting of a perfluorinated tail, link section -SO2-, hydrocarbon chain that can contain amine function and a hydrophilic (water attracting) head group - $\mathrm{COO}-$. The head group of a fluorinated surfactant is either ionic or highly polar giving it the hydrophilic properties, with the most common being anionic groups such as in -COO-, as in $\mathrm{C} 8 \mathrm{~F} 17 \mathrm{SO} 2 \mathrm{~N}(\mathrm{C} 2 \mathrm{H} 5) \mathrm{CH} 2 \mathrm{COOK}$.<smiles>O=S(=O)([O-])C(F)(F)C(F)(F)C(F)(F)C(F)(F)C(F)(F)C(F)(F)C(F)(F)C(F)(F)F</smiles>

Figure 1. C8+S Perfluorooctane sulfonate (PFOS)

It is the perfluorocarbon chain, however, that gives fluorinated surfactants (C8) their unique and highlydesirable property, namely the chain is simultaneously water repellant (that is, it is hydrophobic) and has no attraction for fat (that is, it is lipophobic). No other common surfactant exhibits this distinct property.

\section{Global Environmental Legislation and Action plan for PFOS}

\subsection{European Union - Persistent Organic Pollutants Regulation, 2010(EU-POP)}

- Production, supply and use are now banned with some exemptions.

- Production of Fire-fighting foam with PFOS $\left(\mathrm{C}_{8}\right)$ and above is banned.

- No production of new stocks allowed.

- PFOS-containing foam stocks must be managed as a hazardous waste after June 27,2011.

\subsection{Canada Gazette June 2008}

- As of June 2013 production, supply and use are banned with some exemptions for military use.

- PFOS is specified on the List of Toxic Substances in the Canadian Environmental Protection act, 1999

- Old stocks of PFOS foam to be removed from service in 2013.

- Excess stocks of PFOS foam concentrate can be destroyed by high temperature incineration at any approved hazardous waste destruction facility for a relatively low cost.

\subsection{National Industrial Chemicals Notification and Assessment Scheme (NICNAS) Australia}

- A voluntary phase-out agreement is in existence for PFOS since 2000.

- Recommendations has issued that contain information and advice on the use and handling of PFOS. 
4.4. United States Environmental Protection Agency

- January 26, 2006 announced the "2010/2015 PFOA Stewardship Program,”

- All major surfactant manufactures have agreed on the elimination of these chemicals from emissions and products by 2015 .

\section{AFFF Foams Move into the Future}

It has been more than ten years since $3 \mathrm{M}$ sent a shock wave through the fire protection industry with their announcement that they would end production of PFOS based AFFF foams.This is also about the time that many "experts" said that all AFFF foams would be phased out of production for similar environmental reasons. Instead, telomer-based AFFF foams continue to be the agent of choice to protect against flammable liquid fires, and manufacturers have developed enhanced foam formulations with reduced environmental impacts that can be used well into the future. Why were the experts wrong in their predictions?

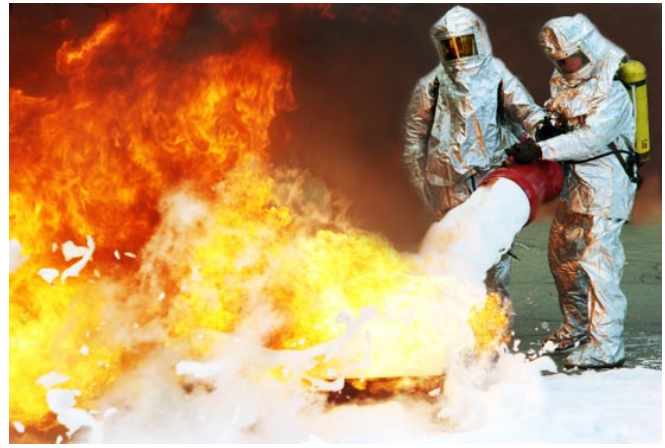

Figure 2. Fighting fire with Foam Applicator

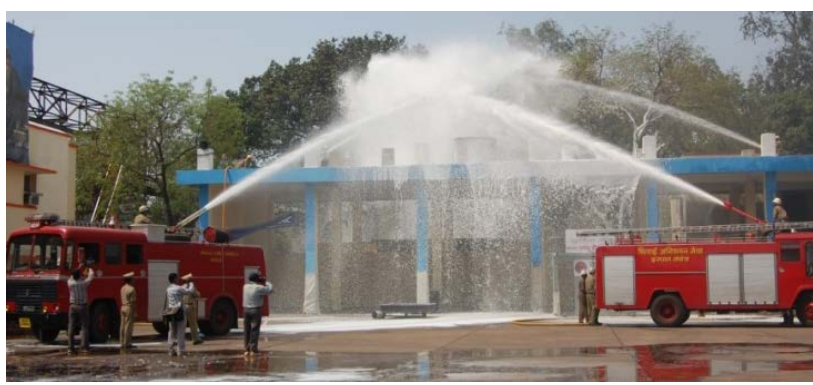

Figure 3. Fighting fire with Foam Applicator

Part of the reason for the speculation about a possible phase-out of AFFF foam after the $3 \mathrm{M}$ announcement was that at the time, the fire protection industry's only real experience with environmental regulation was the phaseout of halons as ozone-depleting substances. Based on this experience many people in the industry assumed that this is how environmental regulation works; a chemical is determined to have some negative impact and is banned from future production. In reality, however, the phase-out of ozone-depleting substances was a unique situation. Most environmental regulation focuses on the toxicity of a substance and has the goals of reducing emissions to the environment and exposure to humans. Very rarely are chemicals actually banned from production. For ozonedepleting substances, the focus was atmospheric effects and it was determined that the only way for the ozone layer to recover fully was to end the worldwide production of these chemicals.

The other reason for speculating about a possible phaseout of AFFF was business related. Companies selling nonfluorosurfactant foams saw an opportunity to use the negative publicity surrounding the phase out announcement as a way to enhance sales of these less effective alternatives. Unfortunately that practice continues today.

In general, there are two approaches to formulate new film-forming foams:

- The first is to identify and synthesize new surfactant molecules that do not contain perfluorinated chains but display the desired film-forming properties.

- An alternative approach is to examine all the properties of fire-fighting foams in order to identify the characteristics that need to be optimized in order to provide the overall suppression performance equivalent to AFFF or FFFP foams, in the absence of the film-forming surfactants.

\section{Fluorine-free Foams}

Fluorine-free fire fighting foams are formulated without using fluorochemicals. To be genuinely fluorine-free the foam concentrate must not contain either fluorosurfactants or fluoropolymers. After 2000, significant developments were made to produce a new generation of fire fighting foams that were fluorine-free. They contain instead watersoluble non-fluorinated polymer additives and increased levels of hydrocarbon detergents. Several types of fluorine-free foams are now available commercially in the market do not have excellent fire fighting performance. Apparently there are foams labeled as fluorine-free which do still contain small amounts of fluorochemicals and are thus not truly fluorine-free; however, the technology of producing 'pure' fluorine-free foams is still evolving.

\section{Fluorotelomer-based Foams}

Fluorosurfactants contained in fire fighting foams have historically been produced from fluorochemicals manufactured by two methods: electrochemical fluorination (ECF) and telomerisation. Foams containing ECF-based fluorosurfactants have not been manufactured in the USA and Europe since 2002 because they contain PFOS (perfluorooctanesulphonate). All modern foams (except some produced in China and India) contain fluorosurfactants produced by telomerisation, usually referred to as fluorotelomers or just telomers. Shorter chain-length $(<\mathrm{C} 6)$ perfluorinated substances are new alternatives for a variety of uses such as textile, carpet and paper additive uses and tile surface treatments and are been reviewed by various agencies regarding their toxicity, bioaccumulation and persistence in the environment and humans. At the same time, however, these new shorter chain length alternatives must not be contaminated significantly with longer chain-length perfluorinated substances of concern during manufacture. These shorter chain length compounds are not relevant for fire fighting foam use as there is a dramatic falloff in fire suppression efficiency with decreasing chain length. 
Over the past several years makers of telomerbased products, not surprisingly, have undertaken more intensive study of the toxicology and environmental fate of their products. For AFFF this research has focused on the predominant breakdown product of the C6 fluorosurfactants they contain, which is commonly referred to as the 6:2 fluorotelomer sulfonate (6:2 FTS). In referring to fluorotelomer sulphonates, the number of fluorocarbons (X) and hydrocarbons (Y) are designated by the abbreviation $\mathrm{X}: \mathrm{Y}$, e.g., 6:2 fluorotelomer sulphonate (6:2 FTS) has six fluorinated carbons and two methylene carbons in the fluoroalkyl chain.This Fluorotelomer-based Foams (6:2 FTS) are having lesser effect on soil \& aquatic life compare to C8 chemistry.

There is no foam with zero effect on the environment. All fire fighting foams whether AFFF-type or fluorinefree have an undesirable effect in the environment to a greater or lesser extent.

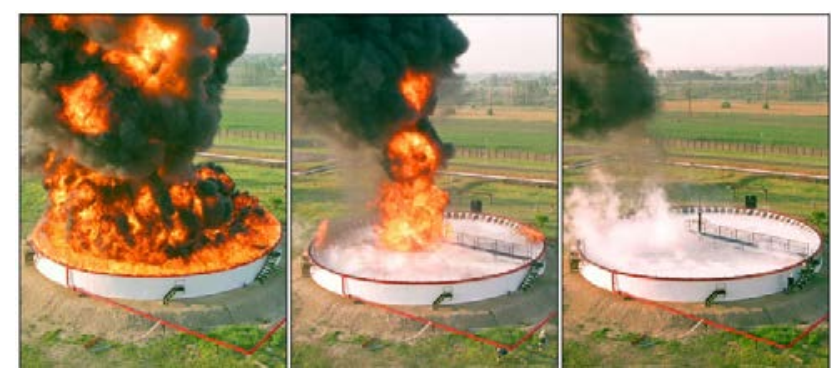

Figure 4. Fighting oil tank fire with Fixed Firefighting Foam System

\section{Conclusion}

The continuous research and development to find out the substitute for perfluorooctanyl sulfonate derivative (C8) has brought two choices i.e. Fluorine-free foams and Fluorotelomer-based Foams. These foams are having low aquatic toxicity and meeting the requirement of different international standers of film forming foam. With this new formulation since more than one decade neither we have achieved $100 \%$ environment friendly or green foam nor have we developed better foam with reference to fire fighting performance. There is every possibility that after 2015 new regulation may come in to effect to restrict the use of these new formulations of AFFF. The particular concern is the use of firefighting foams that have the potential to cause acute, short-term harm as well as some that contain highly persistent chemicals responsible for chronic, long-term impacts. The risks of impacts by foam are not just from the infrequent large-scale incidents such as terminal fires but also the smaller ongoing releases from training activities, accidents, testing and equipment servicing. Hence the objective of our research is to come up with new environment friendly formulation for Aqueous film-forming foams (AFFFs) with high drain time, low bubble coarsening, faster knockdown and excellent burn back resistance.

\section{References}

[1] UL 162, "Standard for Foam Equipment and Liquid Concentrates, "Underwriters Laboratories Inc., Northbrook, IL, Sixth Edition, 1994.

[2] Military Specification, "Fire Extinguishing Agent, Aqueous FilmForming Foam (AFFF) Liquid Concentrate, for Fresh and Seawater,” MIL-F-24385F, 7 January 1992.

[3] Conference review, 2013, Fifth International Fire Fighting Foam Conference, Reebok Stadium, Bolton (UK)

[4] Guruge K.S, Yeung L.W.Y, Li P, Taniyasu S, Yamashita N, Nakamura M. 2011., Fluorinated alkyl compounds including long chain carboxylic acids in wild bird livers from Japan.

[5] Jin C.F., Sun Y.H., Islam A., Qian Y., and Ducatman A., 2011. Perfluoroalkyl acids including perfluorooctane sulphonate and perfluorohexane sulphonate in firefighters Amer. Coll. Occup. Environ. Med.

[6] Aerobic biodegradation studies of 6:2 fluorotelomer sulfonate in activated sludge, $\mathrm{N}$. Wang et al, DuPont, manuscript in preparation, 2010.

[7] Environmental Impacts of Fire Fighting Foams by William H. Rupert, Daniel P. Verdonik, and Christopher Hanauska of Hughes Associates, Inc. (2005)

[8] Serex, T. et al., (2009). Hazard evaluation of 6-2 fluorotelomer alcohol (6-2 FTOH), 1,1,2,2-tetrahydroperfluorooctanol. The Toxicologist, Supplement to Toxicological Sciences.

[9] Liu, J.; et al. (2010). 6-2 Fluorotelomer alcohol aerobic biodegradation in soil and mixed bacterial culture. Chemosphere

[10] Scheffey, J. L., Leach, W., and Fallis, S., "Performance Analysis of Foam Agents Required to Combat Liquid Fuel Hazards," Proceedings of the AFFF and the Environment Working Group, Panama City, FL, Oct. 16-18, 2001.

[11] Dr. Jimmy Seow, Department of Environment and Conservation Western Australia, 2013, Fire Fighting Foam with perfluorochemical environmental review.

[12] Apelberg B.J., Witter F.R., Herbstman J.B., Calafat A.M., Halden R.U., Needham L.L., Goldman L.R., 2007b. Cord serum concentrations of perfluorooctane sulphonate (PFOS) and perfluorooctanoate (PFOA) in relation to weight and size at birth. Environ. Health Perspect. 115(11), 1670-1676.

[13] Australian Department of Defence, June 2007. Environmental Guidelines for Management of Fire Fighting Aqueous Film Forming Foam (AFFF) Products.

[14] Buck R.C., Hoke R.A. and Serex T., 2011. The toxicology of 6:2 Fluorotelomer Sulphonate C6F13CH2CH2SO3-, 6:2 FTSA. 3rd International Workshop on nthropogenic Perfluorinated Compounds in Amsterdam The Netherlands on June 15 to 17, 2011.

[15] Schlummer M., Gruber L., Schmitt A., and Lange F.T., 2008. Single application of fire fighting foam has increased levels of perfluorooctane sulphonate (PFOS) in receiving waters and fish.

[16] Canada Gazette, 2010. Order Adding Toxic Substances to Schedule 1 to the Canadian Environmental Protection Act, 1999. Vol. 144, No. 21, October 13, 2010. 